\title{
Neurotoxins may go unrecognised
}

Damage to the nervous system by industrial chemicals is still an underresearched subject, according to some scientists. This is probably due to old ideas concerning the mode of action of neurotoxins. Until a few years ago, it was assumed that peripheral neuropathy - often characterised by a tingling in the fingers and toes-was one of the earliest signs of nerve toxicity.

The 'dying-back' hypothesis, used to describe the phenomenon, held that toxic derangement of neuronal action began with the loss of metabolic activity at the nerve terminal, followed by progressive failure of the neuron in a retrograde (dying-back) fashion towards the cell body if impairment continues. In addition, it was generally believed that, if intoxication ceased, the nerve would regrow, and the subject would recover. And finally, the hypothesis held that it was the longest and largest diameter axons (supporting the largest metabolic load) which were the most vulnerable to neurotoxins.

These ideas are no longer tenable, according to Drs Herbert Schaumberg and Peter Spencer of the Neurotoxicity Unit of the Albert Einstein College of Medicine in New York. In a seminal paper, delivered at the recent New York Academy of Sciences conference on Public Control of Environmental Health Hazards, Schaumberg argued the case for a reappraisal of the neurotoxic hazard posed by industrial chemicals.

Two of those he and Spencer selected for study were acrylamide monomerthe precursor of acrylamide polymerand a common industrial and laboratory solvent, n-hexane. Using cats as the test species, and the hindpaw as the limb of choice, the two demonstrated that acrylamide not only caused degeneration to regions of the axon prior to an effect on the nerve terminal, but that the most sensitive nerve in the hindlimb was one of 7-11 $\mu \mathrm{m}$ diameter, and not the 11-20 $\mu \mathrm{m}$ diameter axon.

With n-hexane, believed to be neurotoxic after conversion to the 2,5-hexamedione, degeneration in the hindlimb occured in the nerves to the calf muscles prior to those near the foot. And in this case, it was the hindlimb which was attacked before the forelimb. So with n-hexane, Schaumberg believes that axon length and diameter are probably important in determining vulnerability.

In view of these findings, Schaumberg considers the term 'dying-back' to be inappropriate. Too many clinicians associate the expression solely with peripheral changes. Schaumberg and Spencer prefer to describe these pathological processes as "central-peripheral distal axonopathy'.

It is this use of the word 'central' which should cause the regulatory agencies to sit up and take note for it covers a striking and consistent observation made in the course of Schaumberg's studies with n-hexane. At the same time that changes were first noted in the vulnerable areas of the peripheral nervous system (PNS), axonal degeneration had occurred in the central nervous system (CNS). If exposure to the chemical is halted early enough, Schaumberg believes that the nerves may recover; however, if contact is prolonged, a stage is reached beyond which the CNS will not recover, the peripheral nerves being the only ones where regeneration is possible.

In cases of prolonged, low-level exposure to n-hexane, Schaumberg suggests, subtle changes may occur to the nervous system vital to memory and vision. And he adds that as neuronal function and number decline in the course of the normal aging process, individuals exposed to compounds such as n-hexane and acrylamide might experience premature deterioration in vision or mentation.

Schaumberg's investigations of a group of young Italian women exposed to n-hexane illustrate some of the problems in this field. The women, employed in 'cottage-industry' shoe factories near to Naples worked long hours in small, poorly-ventilated rooms. Rags saturated with a solution containing n-hexane were constantly handled in the daily work routine.

In one case, it was two years after inhalation of large amounts of nhexane vapour that one of the women developed symptoms of cramping sensations in the hands, accompanied by weight loss and symptoms of anorexia. Two weeks after onset, the subject experienced cramp in her calves and developed an unsteady gait which was improved by the wearing of highheeled boots. After a further two months, the legs weakened, and toes became numbed. She was finally admitted to hospital after a further two months, when walking to work became an impossibility. According to Schaumberg, two years later she still has a "slow, stiff-legged, waddling gait".

Some of the other women with prolonged high-level exposure to n-hexane have had a more devastating clinical history. Several have been quadraplegic for months; two have required transient respiratory assistance, two others have been left with visual impairment; and one adolescent has experienced a general decline in initiative and general intellectual ability.

The most extraordinary aspect of these cases has been the assumption on the part of an Italian neurologist that the women were suffering from multiple sclerosis. The local general practitioner concerned with the cases refused to accept this prognosis, and Schaumberg was consulted. He pronounced hexane poisoning, and gave evidence to that effect during the course of a compensation suit filed on behalf of the women. The evidence was accepted, and they won their case.

Schaumberg considers that part of the blame for the misdiagnosis must rest with the shortage of research on neurological damage caused by chemical agents. Many of the regulatory agencies are not even aware of the problem, he says. And the one agency which is, the US Environmental Protection Agency, still persists in recommending an insensitive 'demyelination' assay for neurotoxicity studies. This assay, argues Schaumberg, will not detect the early primary axonal changes which eventually result in myelin breakdown.

One drawback encountered with the current tests for neurotoxicity is that, because they are bioassays, they are both time-consuming and costly. Behavioural tests are also employed; but, until the present time, neither type of test has been compared for accuracy. Now, however, a collaborative study, undertaken by the Albert Einstein Neurotoxicity Unit and the National Institute of Environmental Health, is considering simultaneous behavioural and morphological studies of the nervous system at various stages of intoxication in acrylamide-treated rats.

Although this collaboration will validate current screening processes for neurotoxic agents, investigators believe that future tests will be biochemical. Enzyme markers would be the best indicators and the enzymes of the Krebs cycle are currently under scrutiny. The Krebs cycle is viewed as being more promising than monitoring changes in essential fatty acid metabolism. Few neurotoxins--diptheria is one example-attack the myelin sheath and involve fatty acid changes.

A more universal test is still a researcher's dream. For the moment, we do know that chemicals attack the nervous system in a number of subtle ways. Clinical tests will have to be flexible enough to detect a range of pathological symptoms. And it has to be remembered that both the PNS and the CNS are vulnerable to attack. In the case of the former, recovery generally occurs when intoxication ceases; but when the latter is involved, damage may be permanent.

Alastair Hay 